\title{
The effect of bilateral pelvic neurectomy on uterine and abdominal electrical and pressure activity, as measured by telemetry in conscious, unrestrained pregnant rats
}

\author{
Lynnette B. Mackay ${ }^{1}$, Shao Q. Shi ${ }^{2}$, Robert \\ E. Garfield ${ }^{2}$ and William L. Maner ${ }^{2, *}$ \\ 1 University of Texas Medical Branch, USA \\ 2 Department of Obstetrics and Gynecology, \\ St. Joseph's Hospital, Phoenix, AZ, USA
}

\begin{abstract}
Aims: Bilateral pelvic neurectomy (BPN) interferes with delivery in rats. This work measures the effect of BPN on uterine and abdominal electromyography (EMG) and pressure during gestation and delivery.

Methods: Uterine EMG and intra-uterine pressure (IUP), as well as abdominal muscle EMG and intra-abdominal pressure (IAP), were recorded on days 19-24 in rats. Mean values were determined for the peak frequency of the power density spectrum (PDS) of the uterine and abdominal EMG "bursts," the integral of the abdominal EMG power spectrum (IPS), and the area under the IUP and IAP curves (AUIUP and AUIAP).

Results: Uterine EMG power density spectrum peak frequency and AUIUP were low during days 19-21 in all rats, and on days 22-23 increased sharply and significantly in both groups. Before delivery, sham rat recordings showed sudden sharp increases in AUIAP, AUIUP and IPS as "pushing" was observed. Pushing was not observed or recorded in laboring BPN rats and they did not deliver normally.

Conclusions: A functional pelvic nerve is required for normal labor and delivery in rats. BPN inhibits abdominal EMG and contractions necessary to expel the fetus.
\end{abstract}

Keywords: Bilateral pelvic neurectomy; dystocia; EMG; fetus-expulsion-reflex; telemetry.

\section{Introduction}

Pelvic nerves play an important role in successful pregnancy, labor and delivery in rats [6]. Female rats sub-

\footnotetext{
*Corresponding author:

William L. Maner

St. Joseph's Hospital

Obstetrics and Gynecology

825 W. Osborn Road \#4022

Phoenix, AZ, USA

E-mail: William.Maner@chw.edu
}

jected to bilateral pelvic neurectomy (BPN) do not conceive after the procedure. It appears that luteolysis is inhibited, and that they will only become pregnant after treatment with reserpine to induce luteolysis for implantation [15]. Rats that are already pregnant when subjected to BPN are able to maintain pregnancy to term, but do not deliver normally. They appear to go into labor at term, but labor is very prolonged, and most pups are born dead or are retained in utero. This dystocia has been studied by many researchers [4-6, 15, 23-25], including those studying the effects of endocrine [25], gap-junctions [4, 5], pelvic nerves [23, 24], and fetalexpulsion-reflex [15]. The effects of denervation on pregnancy in rats has been studied [7, 12, 16, 19], but no one has attempted to look at contractility with both electrical and mechanical parameters in conscious, unrestrained animals.

We have already ascertained that the cervix ripens despite BPN [21], and considering that progesterone withdrawal is probably close to normal $[4,5]$, we decided to further investigate the BPN phenomenon. In this study, we intended to measure the effect of BPN on uterine and abdominal electromyography (EMG) and intrauterine pressure during gestation and delivery. Our objective was to assess by telemetry these effects of BPN during the course of gestation and delivery in conscious unrestrained rats.

\section{Materials and methods}

Timed-pregnant Sprague Dawley rats $(n=18)$ were received from Charles River Laboratories (Wilmington, MA) early in gestation (around day 11). The animals were housed in separate cages in the animal facility with controlled light cycle (12 h dark/ light), temperature and humidity, and allowed food and water ad libitum.

These rats have a gestation of 22-23 days, day 1 being the day that a positive sperm smear is observed. On day 15 of gestation, the rats were anesthetized with a mixture of Ketamine $(50 \mathrm{mg} / \mathrm{kg})$ and Xylazine $(5-10 \mathrm{mg} / \mathrm{kg})$ injected intra-peritoneally, and laparotomized. In the BPN rats $(n=9)$, the pelvic nerves were retrieved and bilaterally dissected as described in detail elsewhere [6], and in sham control rats $(n=9)$, the nerves were exposed and touched, but left intact.

At the time of the surgery, all animals were outfitted with internal telemetry devices (C50-PXT models from Data Sciences, St. 
Paul, MN) for measuring EMG and pressure. In order to record uterine EMG and intrauterine pressure, the electrodes were sutured to the uterus, and a pressure catheter was sutured into the uterine cavity. Previous studies have demonstrated that this method is effective at measuring uterine EMG and internal pressure, without adverse effects on delivery mortality or morbidity rates [20], as long as the animals are given several days to heal before delivery. For studying abdominal-muscle effects $(n=10)$, the electrodes were sutured internally into the rectus abdominis muscle and the pressure catheter was allowed to move freely in the abdominal cavity to measure the intra-abdominal pressure (IAP). After surgery, the rats were allowed to recover in warmed cages, and were injected sub-cutaneously with buprenorphine $0.1 \mathrm{mg} / \mathrm{kg}$ twice daily for two to three days until recording commenced. All procedures were performed in accordance with the requirements of the Institute of Animal Care at UTMB.

Recordings of EMG and pressure from both abdominal and uterine muscle continued from day 19 until noon of day 24 (term $=d 22 / 23)$, when all rats were sacrificed. Mean values were determined for the power density spectrum (PDS) peak frequency of the uterine and abdominal EMG "bursts", and for the area under the IUP (AUIUP) and IAP (AUAUP) curves, with all parameters averaged over three 2-hour time periods each day. In some cases, the bursts held both abdominal and uterine electrical frequency components (during pushing), and in other cases the bursts held only uterine electrical components (during uterine contractions in non-pushing sham and BPN animals).

Uterine EMG activity was sampled at $10 \mathrm{~Hz}$, and band-pass filtered from $0.3-5 \mathrm{~Hz}$, since our previous studies have shown this low electrical frequency range to be typical for rat uterine muscle $[11,26]$. The EMG activity of the rectus abdominis muscle was sampled at $400 \mathrm{~Hz}$, and band-pass filtered from 0.3 to $200 \mathrm{~Hz}$, since such high frequencies are typically found in striated muscles [18]. Recorded data were transmitted to an external receiver (RLA 1020 model, Data Sciences) and recorder (MacLab 16/s, AD Instruments, Castle Hill, Australia), and stored on a PC. Analysis of the data was performed using Chart 4.0 software (AD Instruments, Castle Hill, Australia) which applied a Fourier transformation to the uterine electrical data in order to calculate the PDS of each uterine electrical "burst" associated with each contraction, and to locate the frequency of the largest power peak in the 1.0-5.0 Hz range (uterine-specific frequency for rats - $[11,26]$. From these data we calculated mean values for the peak frequency of the PDS for both the uterine and abdominal EMG bursts $(\mathrm{Hz})$, the integral of the abdominal EMG power spectrum (IPS, an estimate of the electrical energy associated with an abdominal contraction, which was found predominantly in the $50.0-150.0 \mathrm{~Hz}$ frequency range), and calculated the area under the IUP and IAP curves (AUIUP and AUIAP; units are in $\mathrm{mm} \mathrm{Hg} \times \mathrm{s}$ ).

Student's $t$-test and one-way ANOVA methods were used for statistical analysis (significance $\mathrm{P}<0.05$ ).

\section{Results}

Sham rats proceeded to deliver normally at term. Cervical distention was observed in all animals, and most BPN rats had vaginal bleeding at term, indicating cervical ripening. After a prolonged labor, one or two dead pups were sometimes delivered from the BPN animals. However, complete, normal delivery was never achieved in any of the BPN animals.

Uterine pressure and EMG traces showed uterine contractile activity superposed with spikes of activity associated with pushing from the abdominal muscles (Figure 1). These abdominal pressure spikes often occurred right at, or near to, the top of the uterine pressure curves. Uterine EMG peak frequency and IUP activity was relatively low during gestational days 19-21 in both sham vs. BPN rats $(1.31 \pm 0.09 \mathrm{~Hz}$ vs. $1.38 \pm 0.06 \mathrm{~Hz}$, respectively, and $54.2 \pm 3.66 \mathrm{~mm} \mathrm{Hg} \times \mathrm{s}$ vs. $44.0 \pm 8.26 \mathrm{~mm}$ $\mathrm{Hg} \times \mathrm{s}$, respectively - Figure 2A,B). On days 22-23 in laboring rats, the mean PDS peak frequency of the uterine electrical bursts increased sharply and significantly (compared to day 19-21 levels) in both sham and BPN animals, to $2.59 \pm 0.11 \mathrm{~Hz}$ and $2.44 \pm 0.10 \mathrm{~Hz}$, respectively. AUIUP also increased significantly (compared to day 19-21 levels) in both sham and BPN animals to $198.9 \pm 34.4 \mathrm{~mm} \mathrm{Hg} \times \mathrm{s}$ and $239 \pm 46.25 \mathrm{~mm} \mathrm{Hg} \times \mathrm{s}$, respectively (Figure $2 A, B$ ). Upon delivery in sham rats (i.e., $<1 \mathrm{~h}$ before the first pup was delivered), uterine EMG PDS peak frequency achieved its highest value in both sham and BPN animals (to $3.64 \pm 0.12 \mathrm{~Hz}$ and to $3.57 \pm 0.27 \mathrm{~Hz}$, respectively). During actual delivery, sham animals AUIUP showed maximal values (305.65 \pm $52.27 \mathrm{~mm} \mathrm{Hg} \times \mathrm{s})$ vs. BPN animals (193.19 $\pm 18.94 \mathrm{~mm}$ $\mathrm{Hg} \times \mathrm{s}$ ). During this initial delivery period, AUIUP was significantly higher in sham animals compared to BPN animals.

AUIAP changes from baseline, measured on days 19-21 in sham and BPN rats, were small and insignificant $(5.02 \pm 8.61 \mathrm{~mm} \mathrm{Hg} \times \mathrm{s}$ and $2.1 \pm 4.9 \mathrm{~mm} \mathrm{Hg} \times \mathrm{s}$, respectively). At day 22-23, sham, but not BPN rats, engaged in the very distinctive activities of pushing during labor, with a significant increase in AUIAP (55.1 $8.35 \mathrm{~mm} \mathrm{Hg} \times \mathrm{s}$ for shams vs. $6.1 \pm 0.71 \mathrm{~mm} \mathrm{Hg} \times \mathrm{s}$ for BPN). Bursts of activity were interspersed with quiescent periods, and pressure spikes, due to abdominal pushing were also present in recordings of uterine contractions in sham, but not in BPN, animals (Figure 3). These pressure spikes (about 5-10 s in length - usually 2-4 per uterine contraction), increased the total AUIUP by $26.35 \pm 4.5 \%$. The inter-abdominal cavity measurements also showed a difference in pressure for pushing and non-pushing animals. AUIAP was significantly higher in sham animals that pushed as compared to BPN animals that never pushed (Figure 4).

During term parturition in sham rats, power spectra of EMG bursts recorded included both uterine $(<5 \mathrm{~Hz})$, as well as abdominal (50-150 Hz range) electrical frequency components only in pushing animals (Figure 5A). However, during all uterine contraction periods for BPN animals, and for some sham non-pushing contraction intervals (only a few such events observed near delivery), only uterine activity was found in the bursts (Figure 5B). 

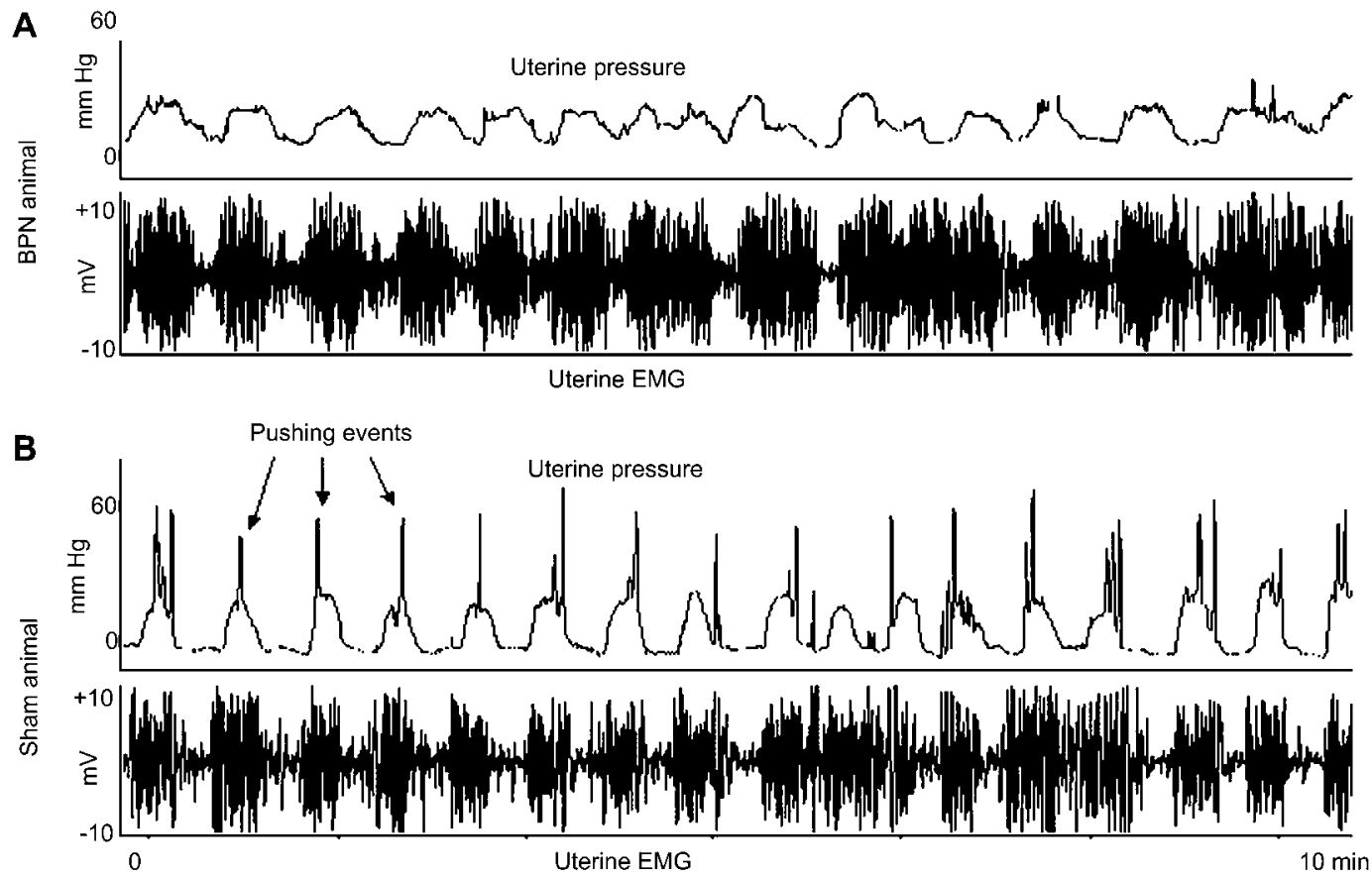

Figure 1 Recording of uterine EMG bursts (bottom traces - which contain uterine and abdominal electrical components) and uterine contraction pressure (top traces) in a neurectomized (BPN) rat in labor (A), but which is unable to push to assist with the delivery process, as compared to a sham rat (B) also in labor and which is pushing. Notice the pressure spikes (arrows), which indicate pushing events in the sham animal, superposed on the uterine contraction traces.

Mean values were determined for the integral of the burst power spectrum of the EMG bursts. IPS was significantly greater in electrical bursts for the sham group when pushing $\left(26.92 \pm 14.07 \mathrm{~V}^{2} \times \mathrm{Hz} \times 10^{-5}\right)$, as compared with IPS of non-pushing electrical bursts from the same sham animals $\left(1.20 \pm 0.61 \mathrm{~V}^{2} \times \mathrm{Hz} \times 10^{-5}\right)$, and as compared with the IPS of electrical bursts in the BPN group $\left(0.79 \pm 0.12 \mathrm{~V}^{2} \times \mathrm{Hz} \times 10^{-5}\right)$, the animals of which never pushed (Figure 6). There was no significant difference between IPS of sham non-pushing bursts vs. BPN
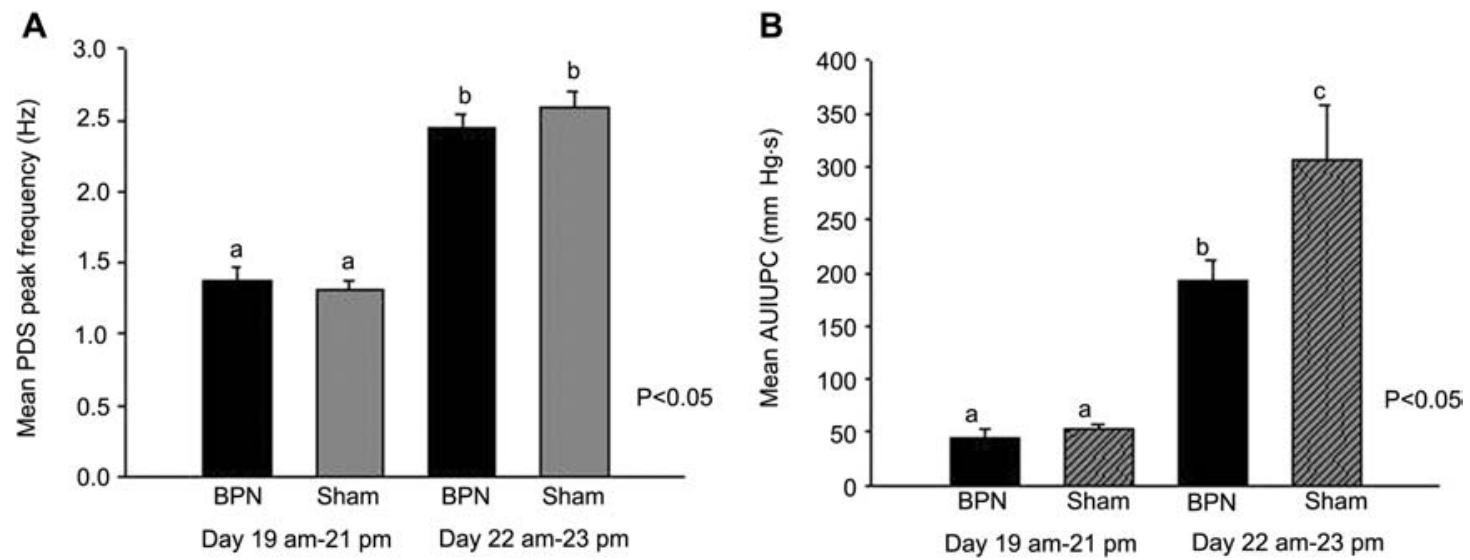

Figure 2 Mean values \pm Standard Error for uterine power density spectrum (PDS) peak frequency (A) and area under intrauterine pressure AUIUP (B) on days 19-21 (non-labor) compared with days 22-23 (labor) for neurectomized (BPN) and sham controls. Note that mean values with different letters above the bars show significant differences $(P<0.05)$. The uterine EMG and pressure are significantly greater in the laboring sham and BPN animals, compared to the non-labor animals, which means that uterine activity is normal in BPN animals. However, BPN animals do not deliver their pups, and this suggests that lack of abdominal muscle activity during delivery is the cause. During actual delivery, AUIUP value reaches significantly higher levels in BPN animals compared to sham animals, due to the pushing activity. 

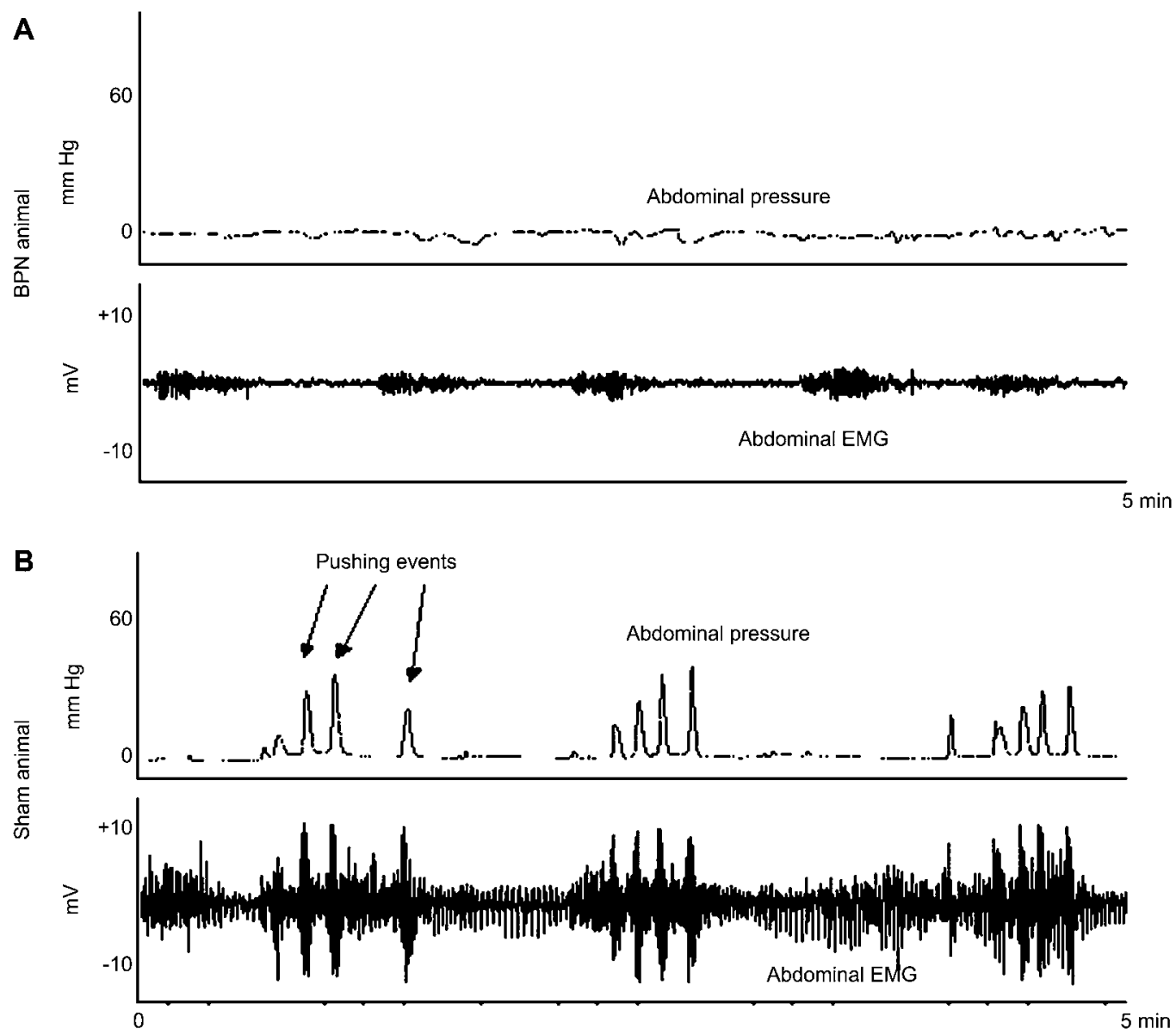

Figure 3 Recording of EMG bursts (bottom traces - which contain both uterine and abdominal electrical components) and abdominal pressure contraction events (top traces) in a BPN rat in labor (A), but which is unable to push to assist with the delivery process, as compared to a sham rat (B) also in labor and which is pushing. Notice the prominent abdominal pressure spikes (arrows) associated with pushing in the sham animal. These pressure spikes make the difference between successful and failed delivery in animals that have normal uterine contractile activity.

(always non-pushing) bursts, which essentially contained nothing but background noise. This was largely due to the fact that the uterine portion of the electrical signals was filtered out from this analysis, leaving only abdominal muscle frequencies, and the sham non-pushing intervals during contractions, as well as the BPN contraction intervals, were both nearly completely devoid of abdominal muscle electrical activity.

\section{Discussion}

Our aim was to further investigate the changes in uterine activity and the role of abdominal muscle EMG and contractility in the labor process of term rats after BPN with inhibited delivery. We recorded uterine and abdominal activity and were able to analyze PDS data from electrical bursts from both the uterine smooth and the abdominal striated muscle. By comparing the integrals of the power spectrum of the abdominal muscle bursts, we assessed that during abdominal contractions (which occurred as the rats were pushing) there was a significantly higher amount of muscle electrical energy for sham animals, but that there was no significant difference in these parameters between non-pushing sham bursts and BPN bursts, which were both significantly low.

Until day 21 of gestation, in sham and neurectomized rats, the uterine EMG was generally erratic and showed few bursts with low frequency. Close to term, bursts became more frequent, longer, of greater amplitude and higher frequency. From $1 \mathrm{~h}$ to $1 / 2 \mathrm{~h}$ before the first pup was delivered to the sham rats, they were "pushing", and pressure peaks were recorded simultaneously in the abdominal and uterine probes, superimposed on uterine contraction peaks (Figures 1 and 3). In fact, the abdominally generated activity almost always occurred at the very peak point along the uterine contraction activity. This is indirect evidence that the abdominal activity is, in fact, an involuntary effect triggered by a feedback mechanism associated with the uterine pressure on pelvic ner- 


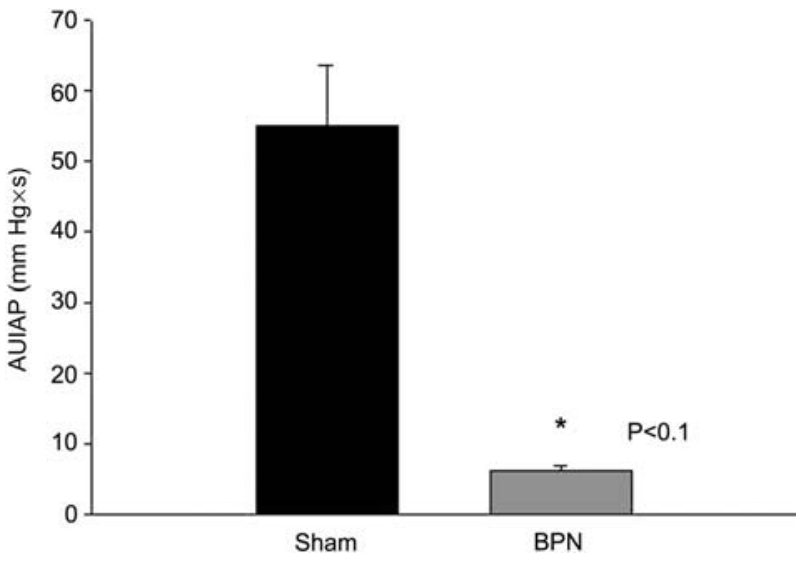

Figure 4 Area under intra-abdominal pressure (AUIAP) for sham pushing rats is significantly higher than for neurectomized (BPN) rats (mean \pm Standard Error shown), which cannot, or do not, push during delivery. These additional abdominal pressure spikes (which translate into additional intrauterine pressure spikes - see Figure 1), as well as the associated positional "crunching" of the mother, help pass the pups past the pelvic region and successfully out of the vaginal canal.

ves. In the BPN rats, such "pushing" was not observed, and the abdominal and uterine pressure records did not show these extra spikes.

Both sham and BPN animals displayed uterine-specific electrical frequencies and pressures normal for labor [11, 26], so outcome differences in delivery are attributable, in part, to the abdominal muscle contribution to the delivery process. Studies have shown that effective pushing is an essential part of successful delivery in women [3]. Without effective pushing, the probability of operative delivery is much higher due to maternal exhaustion and fetal distress after a prolonged second stage. Delivery of a baby with dystocia can be facilitated by changing the angle of the birth canal using McRobert's position [2]. This also promotes increases in the uterine pressure to more readily push the fetus.

From the present study, we suggest that cervical function played no part in the inability of the BPN animals to deliver [21]. The BPN animals experienced loss of the pushing reflex, which stimulates abdominal muscle contractions. This is because the afferent nerves present in the pelvic nerve are required for this reflex $[9,13]$. We surmise that since the animal receives no feedback from cervical and vaginal extension, it cannot be stimulated to push for proper delivery of the pups, and is not observed to crouch and sometimes not to even bleed vaginally. Because of this, the fetuses may not be properly 'assisted in passage' of the birth canal by the position of the mother. Since such crouching and bleeding is always observed in normal rat delivery, it seems to be an essential part of the delivery process. At necropsy, the pups are grouped at the cervical end of the uterus with placentas detached, suggesting that the uterus has produced
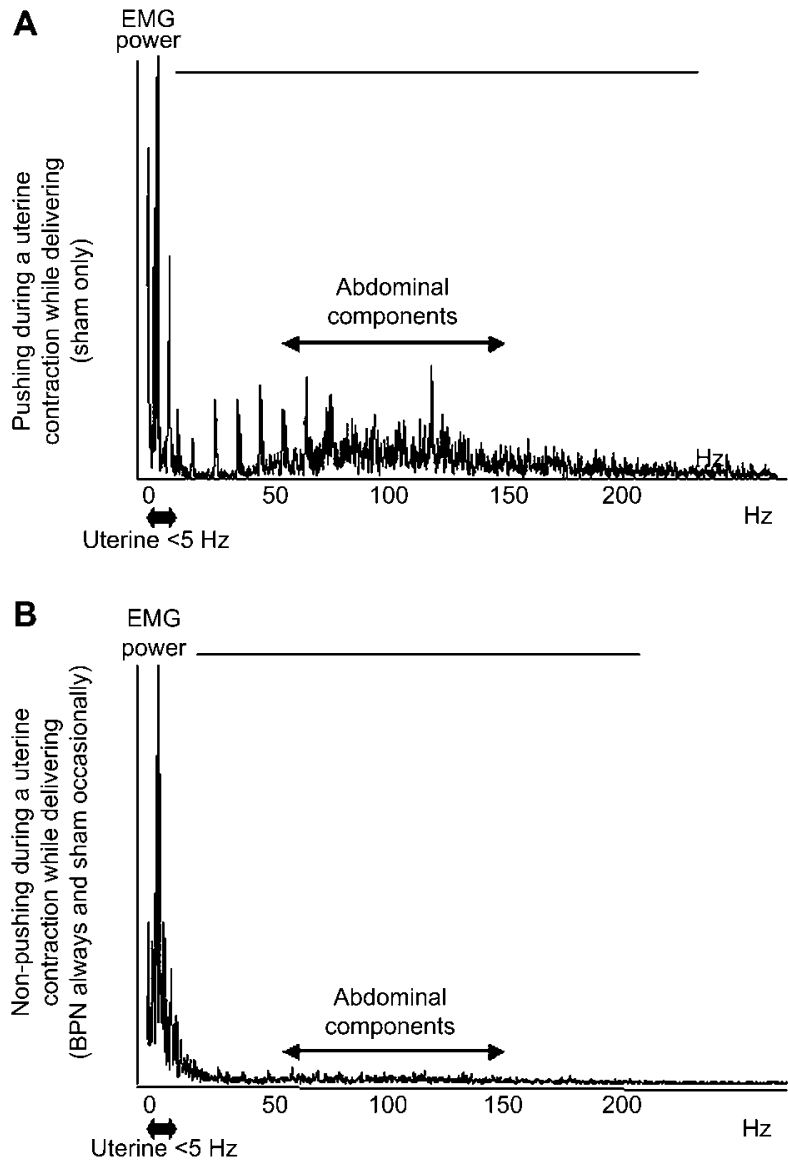

Figure 5 Power density spectrum of EMG burst (A) from a sham rat during uterine contraction while pushing - containing uterine + abdominal electrical frequencies, $<5 \mathrm{~Hz}+50-150 \mathrm{~Hz}$, and $(\mathrm{B})$ from a neurectomized (BPN) rat during a uterine contraction when unable to push - containing uterine-only electrical frequencies, $<5 \mathrm{~Hz}$. Note that these type of power spectra were used in calculating the integral of the power spectrum IPS values. It is important to realize that a comparison of uterine electrical activity between BPN and sham animals shows frequencies and amplitudes that are very similar, while that of the abdominal activity is quite different.

strong contractions, but that the fetuses are unable to progress beyond the pelvic barrier. This is further supported by previous studies of neurectomized rats that were treated to increase oxytocin levels, and in which there were increased uterine contractions (as expected), but also in which the animals were still unable to deliver [28].

In rats, and to a lesser degree in humans, the involuntary stretching/crunching/pushing efforts and actions that are corresponding with the labor and delivery process do give rise to a whole host of changes in pelvic, bodily, and vaginal relative positioning, and thus also cause changes in the birth canal angle. Many different labor positions have been observed and measured for their effectiveness in humans [14], and significant differences do exist between them insofar as delivery outcome 


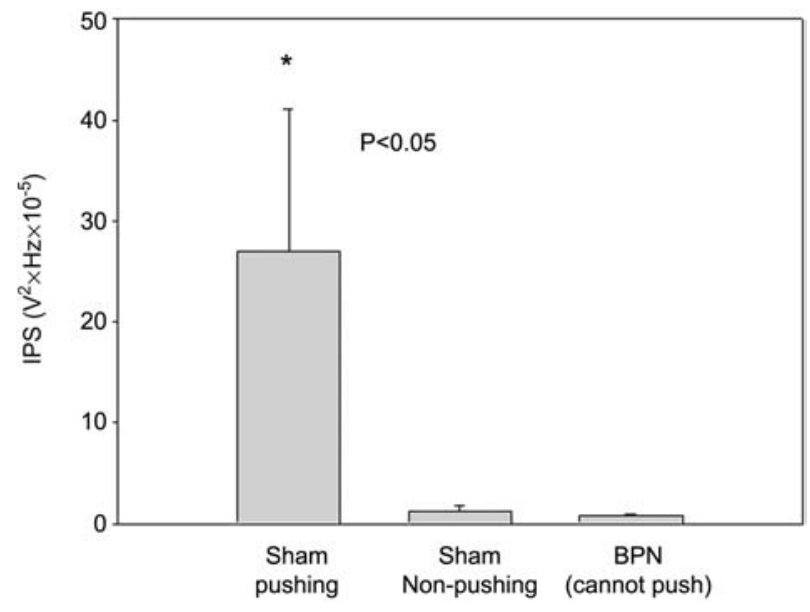

Figure 6 Integral of the power spectrum (IPS) was significantly greater in electrical bursts for the sham animals when pushing (26.92 $\left.\pm 14.07 \mathrm{~V}^{2} \times \mathrm{Hz}^{2} \times 10^{-5}\right)$, as compared with IPS of nonpushing electrical bursts from the same sham animals $\left(1.2 \pm 0.61 \mathrm{~V}^{2} \times \mathrm{Hz} \times 10^{-5}\right)$, and as compared with the IPS of all electrical bursts in the neurectomized (BPN) group $\left(0.79 \pm 0.12 \mathrm{~V}^{2} \times \mathrm{Hz} \times 10^{-5}\right)$, the animals of which never pushed during labor or any other time. Mean \pm Standard Error shown.

is concerned. Without the assistance of a pushing reflex, the animals just lay essentially motionless throughout labor, with position, birth canal angle, and pushing that are all completely irregular, and this clearly acts against a successful delivery capability.

As related further to humans, it is well known that epidural anesthesia during labor causes loss of sensation [3] that may lead to prolonged labor [29], possibly as a result of ineffective pushing which might threaten, directly or indirectly, the mother and fetus [1, 17]. This may also happen in women with spinal cord injuries or in those with paraplegia or other similar spinal or nervous system disorders [8]. Other injured or incapacitated patients may also be affected by an inability to push sufficiently during labor. In all of these cases, it would be useful to know quantitatively just how effective is the pushing for the patients. This information may allow the physician to determine who is likely to have a prolonged labor.

One way to accomplish this would be to use intrauterine pressure measurements, but invasiveness and the potential for infection are always drawbacks to this approach. A more preferable method would be to use the non-invasive trans-abdominal EMG which measures uterine contractile activity [22] as well as abdominal and intercostal muscle EMG [10] to assess the effectiveness of "bearing down" efforts. These electrical signals could be quantified (using signal amplitude, action potential frequency, power spectrum, etc.) to give the clinician a measure of the pushing or Valsalva efforts, as well as the effects due to muscle fatigue [27].

\section{References}

[1] Altman MR, Lydon-Rochelle MT. Prolonged second stage of labor and risk of adverse maternal and perinatal outcomes: a systematic review. Birth. 2006;33:315-22.

[2] Buhimschi CS, Buhimschi IA, Malinow A, Weiner CP. Use of McRoberts' position during delivery and increase in pushing efficiency. Lancet. 2001;358:470-1.

[3] Buhimschi CS, Buhimschi IA, Malinow AM, Kopelman JN, Weiner CP. Pushing in labor: performance and not endurance. Am J Obstet Gynecol. 2002;186:1339-44.

[4] Burden H, Capps M, Lawrence I. Gap junctions in the myometrium of pelvic-neurectomized rats with blocked parturition. Am J Anat. 1979;56:105-14.

[5] Burden HW, Louis TM, Lawrence Jr IE. Lack of effect of unilateral pelvic neurectomy on parturition and luteolysis in the rat. IRCS Med Sci. 1980;8:166-7.

[6] Carlson RR, De Feo VJ. Role of the pelvic nerve vs. the abdominal sympathetic nerves in the reproductive function of the female rat. Endocrinology. 1965;77:1014-22.

[7] Collins JJ, Usip S, McCarson KE, Papka RE. Sensory nerves and neuropeptides in uterine cervical ripening. Peptides. 2002;23:167-83.

[8] Cross LL, Meythaler JM, Tuel SM, Cross AL. Pregnancy, labor and delivery post spinal cord injury. Paraplegia. 1992;30:890-902.

[9] Cunningham ST, Steinman JL, Whipple B, Mayer AD, Komisaruk BR. Differential roles of hypogastric and pelvic nerves in the analgesic and motoric effects of vaginocervical stimulation in rats. Brain Res. 1991;559:337-43.

[10] Demaria F, Porcher R, Ismael SS, Amarenco G, Fritel X, Madelenat $P$, et al. Using intercostal muscle EMG to quantify maternal expulsive efforts during vaginal delivery: a pilot study. Neurourol Urodyn. 2004;23:675-8; discussion 679.

[11] Doret M, Bukowski R, Longo M, Maul H, Maner WL, Garfield RE, et al. Uterine electromyography characteristics for early diagnosis of mifepristone-induced preterm labor. Obstet Gynecol. 2005;105:822-30.

[12] Garfield RE. Structural studies of innervation on nonpregnant rat uterus. Am J Physiol. 1986;251:C41-54.

[13] Gintzler AR, Komisaruk BR. Analgesia is produced by uterocervical mechanostimulation in rats: roles of afferent nerves and implications for analgesia of pregnancy and parturition. Brain Res. 1991;566:299-302.

[14] Gupta JK, Nikodem VC. Woman's position during second stage of labour. Cochrane Database Syst Rev. 2000;(2): CD002006.

[15] Higuchi T, Uchide K, Honda K, Negoro H. Pelvic neurectomy abolishes the fetus-expulsion reflex and induces dystocia in the rat. Exp Neurol. 1987;96:443-55.

[16] Hollingswoth $M$. The innervation of the rat cervix and its pharmacology in vitro and in vivo. Br J Pharmacol. 1974; 52:539-47.

[17] Johnson JH, Figueroa R, Garry D, Elimian A, Maulik D. Immediate maternal and neonatal effects of forceps and vacuum-assisted deliveries. Obstet Gynecol. 2004;103: 513-8.

[18] Kaegi S, Schwab ME, Dietz V, Fouad K. Electromyographic activity associated with spontaneous functional recovery after spinal cord injury in rats. Eur J Neurosci. 2002;16: 249-58 
[19] Klucovits A, Gaspar R, Santha P, Jansco G, Falkay G. Functional and histochemical characterization of a uterine adrenergic denervation process in pregnant rats. Biol Reprod. 2002;67:1013-7.

[20] Kothari TH, Maner WL, Shi SQ, Garfield RE, Chen JD. Inhibitory effects of electrical stimulation on delivery in pregnant rats. Eur J Obstet Gynecol Reprod Biol. 2008; 141:18-22.

[21] MacKay LB, Shi L, Maul H, Maner WL, Garfield RE. The effect of bilateral pelvic neurectomy on cervical ripening in pregnant rats. J Perinat Med. 2009;37:263-9.

[22] Maner WL, Garfield RE, Maul H, Olson G, Saade G. Predicting term and preterm delivery with transabdominal uterine electromyography. Obstet Gynecol. 2003;101: 1254-60.

[23] Martínez-Gómez M, Cruz Y, Pacheco P, Aguilar-Roblero R, Hudson R. The sensory but not muscular pelvic nerve branch is necessary for parturition in the rat. Physiol Behavior. 1998;63:929-32.

[24] Peters LC, Kristal MB, Komisaruk BR. Sensory innervation of the external and internal genitalia of the female rat. Brain Res. 1987;408:199-204.

[25] Renegar RH, Steel M, Burden HW, Hodson CA. Endocrine parameters associated with disruption of parturition after bilateral pelvic neurectomy. P Soc Exp Biol Med. 1992; 201:28-33.

[26] Shi SQ, Maner WL, Mackay LB, Garfield RE. Identification of term and preterm labor in rats using artificial neural networks on uterine electromyography signals. Am J Obstet Gynecol. 2008;198:235.e1-4.

[27] Thomas K, Lee RY. Fatigue of abdominal and paraspinal muscles during sustained loading of the trunk in the coronal plane. Arch Phys Med Rehabil. 2000;81:916-20.

[28] Way SA, Douglas AJ, Dye S, Bicknell RJ, Leng G, Russell JA. Endogenous opioid regulation of oxytocin release during parturition is reduced in ovariectomized rats. $\mathrm{J}$ Endocrinol. 1993;138:13-22.

[29] Wittels B. Semin Perinatol. Does epidural anesthesia affect the course of labor and delivery? 1991;15:358-67.

The authors stated that there are no conflicts of interest regarding the publication of this article.

Received August 22, 2008. Revised December 12, 2008. Accepted January 29, 2009. Previously published online March 17, 2009. 